\title{
Dehazing Algorithm Using Wavelength Compensation to Improve Visibility in Foggy Images
}

\author{
Sreedhar Rajput \\ M.Tech Student, Dept. of ECE, MITS, Madanapalle, Andhra Pradesh, India
}

\begin{abstract}
Images of outdoor scenes caught in adverse climatic conditions. Confined light sources frequently come about because of actuation of road lights and vehicle headlights in these conditions. Conventional art restoration strategies can't work with hazy images that included confined light sources and process is very delay. Therefore, a new method proposed which enhances the road scenes hazy images using wavelength compensation and de hazing algorithm. This algorithm worked based on the dark channel prior and depth map estimation. In contrast based techniques better visual perception of hazy images is accomplished by restoring the local contrast of hazy images. Here, the clarity of hazy images is increase by physically based techniques.. Atmospheric light and transmission map components are utilized to recover the scene objects from the degradation.
\end{abstract}

Keywords: color shift, dark channel prior, localized light, haze removal.

\section{INTRODUCTION}

The visibility of images captured in outdoor road scenes can frequently experience degradation as a result of phenomena which include absorption and scattering of the light by the atmospheric particles such as haze, fog, mist, and so on. Related to image visibility degradation can be problematic to many systems which operate under a wide range of weather conditions, including outdoor object recognition systems, remote sensing systems, intelligent transportation systems such as travelling vehicle data recorders and traffic surveillance systems. The amount of absorption and scattering depends on the depth of the scene between a traffic camera and a scene point. So, scene depth information is important for recovering scene radiance in images of hazy environments. These techniques can be divided into two groups given depth and unknown depth. Given depth works based on the assumption that the depth is given [2], this is then used by these approaches to restore hazy images. However, given depth approaches are not suitable for visibility restoration in real world applications due to a serious limitation: the depth information needs to be provided by the user. Unknown depth estimates and there by recover scene radiance $s$ in hazy images. These approaches use either multiple images [6] and or single image. Wang, Bo-Hao Chen, and Yi-Jui Cheng proposed an algorithm [1] which works on the hazy images of single color such as grey images only.

However, these techniques usually require the use of additional hardware devices and demand more computational complexity.

The presences of atmospheric light source add a luminance in area of the hazy image and often causes over compensation. In low complexity wavelength compensation and image de hazing algorithm a method is developed to enhance hazy images, by compensating the attenuation discrepancy along propagation path, and the possible presence is taken into the atmospheric light consideration.

\section{EXISTING METHODS}

The processing of hazy images focuses solely on compensating either light scattering or color change distortion. Techniques are targeting on removal of light scattering distortion includes image de hazing to restore the clarity of the hazy images. Color change correction techniques estimate the mazy image environmental parameters by performing color registration with consideration of light attenuation. An employing histogram equalization in both RGB and HIS color spaces to balance the luminance distribution of color and dynamically mixing the illumination of an object in a distance dependent way by using controllable multicolor light source to compensated color loss. A systematic approach is needed to take all the factors concerning light scattering, color change and possible presence of artificial light source into consideration. The low complexity wavelength compensation and de hazing algorithm, expensive optical instruments or stereo image pairs are no longer required and this algorithm requires less computational resources and complexity is reduced when compared to conventional Wavelength Compensation and Image De hazing algorithm.

\section{PROPOSED METHOD}

We present an effective approach for the haze removal of single images captured during different environmental conditions that not only avoids the generation of artifact effects but also recovers true color. In this method involves three proposed modules, i.e., DCP module [3], a CA module and VR module. 
The DCP module we are used in low complexity wavelength compensation and image de hazing algorithm first the distances between scenes objects to camera is estimated by using a low complexity dark channel prior algorithm. Based on depth map derived the foreground and background area within image is segmented .The light intensities of fore ground and background are the compared, to determine whether an atmospheric light is employed during the image acquiring process. An artificial light is detected; the added luminance is to be eliminated.

CA module recovers the true color of the scenes featuring wide range of weather conditions [7].This CA module determines the intensity statistics for the RGB color space of a captured image in order to acquire the color information.

The last step of our process, the proposed VR module recovers a high quality free image. The below fig1 block diagram shows the proposed technique.

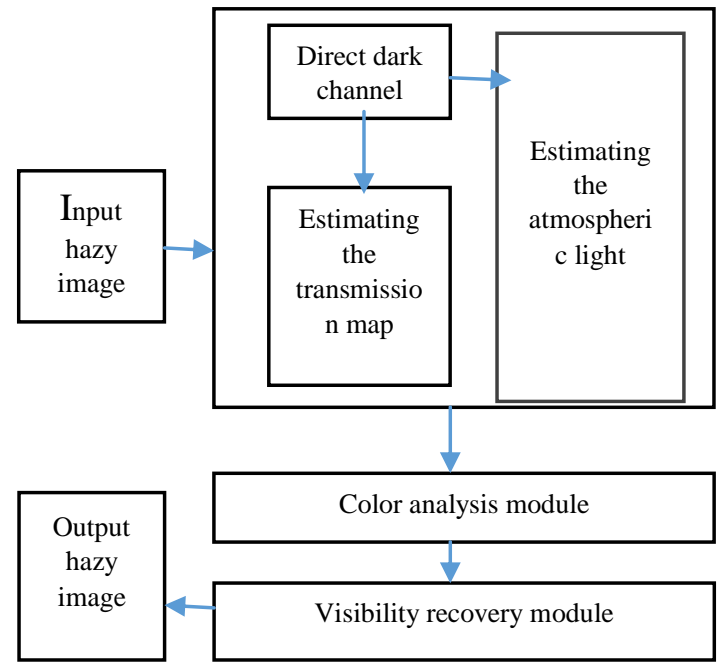

Fig1: Proposed technique

1. Dark channel prior algorithm: The dark channel prior is an existing scene depth derivation method [4], based on the fact that, in most of the background light patches on a haze free image, at least one color channel has a very low intensity at some pixels. The minimum intensity in such a patch should have a very low value called a dark channel. Pixels with a very low value cannot be found in the local patch, which implies the existence of haze. The concentration of haze can be quantified by dark channel prior algorithm. It is turn provides the object camera distance, i.e., the depth map

The hazy image can be modeled by Radiative Transport equation

$$
\mathrm{I}_{\lambda}(\mathrm{x})=\mathrm{j}_{\lambda}(\mathrm{x}) \cdot \mathrm{t}_{\lambda}(\mathrm{x})+\left(1-\mathrm{t}_{\lambda}(\mathrm{x})\right) \mathrm{B}_{\lambda}
$$

Where $\lambda \in\{$ red, green, blue

The dark channel can be calculated by using the equation

$$
\text { Dark channel }=\min \left(\mathrm{I}_{\lambda}(\mathrm{x})\right)
$$

Where $\lambda \in$ \{red, green, blue

$\mathrm{I}_{\lambda}(\mathrm{x})$ is the hazy image acquired by camera. The background light $\mathrm{B}_{\lambda}$ is usually assumed to be the pixel intensity with the highest brightness value in an image.
The brightest pixel value among all local minima corresponds to the background light follows.

$$
\mathrm{B}_{\lambda}=\max \left(\min \left(\mathrm{I}_{\lambda}(\mathrm{x})\right)\right)
$$

The depth map can be calculated by using the formula

$$
\text { Depth map }=1-\min \left\{\operatorname{median}\left(\mathrm{I}_{\lambda}(\mathrm{x})\right) / \mathrm{B}_{\lambda}\right\}
$$

The median filter is used to reduce the block effect 2. Image segmentation: The foreground and background areas of the hazy image are segmented based on the depth map derived using threshold.

$$
\begin{aligned}
& \text { Foreground if } \mathrm{d}(\mathrm{x})>\text { threshold } \\
& \text { Background if } \mathrm{d}(\mathrm{x}) \leq \text { threshold }
\end{aligned}
$$

3. Determination of atmospheric light:

An atmospheric light can be determined by comparing the difference between the mean luminance of the foreground and background images. Higher mean luminance in the foreground indicates the existence of a supplementary light source.

4. Removal of atmospheric light: For removing the atmospheric light, first the average intensity of both the foreground and background image is calculated .Then take the difference between foreground and background intensity. The next step find the luminosity updated foreground intensity is find out by subtracting the difference intensity from the foreground intensity. Then by adding the updated foreground with the background the image free of atmospheric is obtained.

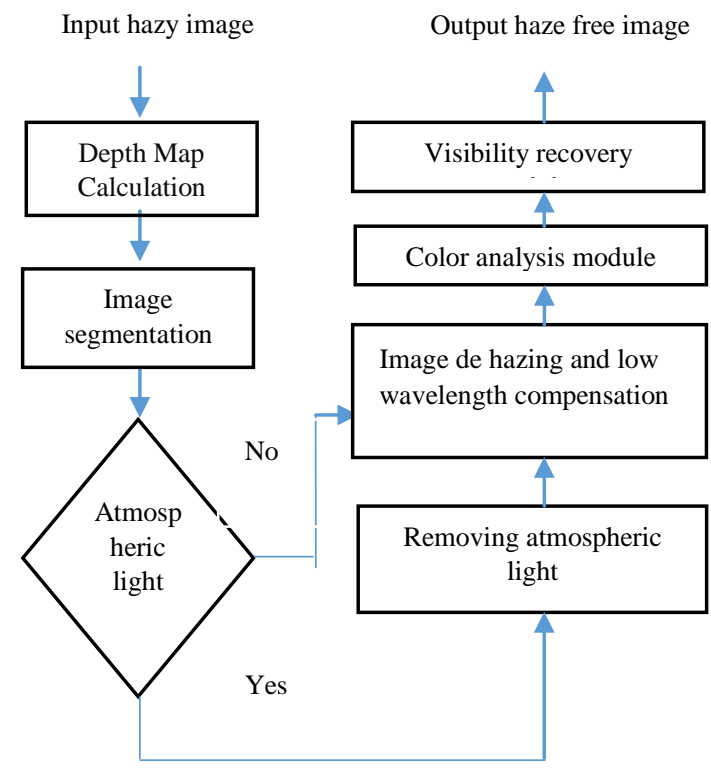

Fig2: Flow chart of WCID algorithm

5. Compensation of light scattering and color change: After removing the atmospheric light source and deriving distance between an object and the camera, the haze can be removed by subtracting the subtracting the in scattering (1-Nrer $\left.{ }^{d(x)}\right) B \lambda$ from image perceived by the camera.

6. Color analysis module: The particles of sand in the atmosphere caused by sandstorms absorb specific Portions 


\section{International Journal of Advanced Research in Computer and Communication Engineering}

Vol. 5, Issue 1, January 2016

of the color spectrum. This phenomenon leads to color shifts in image captured during such conditions, resulting in different color channel distributions. The dark channel prior method uses the same formula for color channel when recovering scene radiance, thereby causing serious color channel shifts in restored images. To solve this problem we propose the CA module that is based on the gray world assumption. This assumption relies on the notion that average intensities should be equal in each RGB color channel for atypical image, which is described as

$$
\begin{aligned}
& R_{\text {avg }}=\frac{1}{M N} \sum_{\substack{x=1 \\
y=1}}^{y=1} \sum_{\substack{x=1 \\
y=1}} I^{r}(x, y) \\
& G_{\text {avg }}=\frac{1}{M N} \sum_{\substack{x=1 \\
y=1}} \sum_{\substack{x=1 \\
y=1}}^{g}(x, y) \\
& B_{\text {avg }}=\frac{1}{M N} \sum_{x=1} \sum_{x=1} I^{b}(x, y)
\end{aligned}
$$

$\operatorname{Where}^{\mathrm{r}}(\mathrm{x}, \mathrm{y}), \mathrm{I}^{\mathrm{g}}(\mathrm{x}, \mathrm{y})$, and $\mathrm{I}^{\mathrm{b}}(\mathrm{x}, \mathrm{y})$ represent the captured image in the RGB color channels respectively, and $\mathrm{MN}$ represents the total number of pixels in the captured image.Based on this assumption ,color spectrum adjust parameter can be produced for each RGB color channel in order to avoid color shifts in the restored image.This can be measured as

$$
\gamma^{C}=\frac{\frac{1}{\mathrm{MN}} \sum_{\mathrm{x}=1}^{\mathrm{y}=1} \sum_{\mathrm{x}=1}^{\mathrm{y}=1} \mathrm{I}^{\mathrm{b}}(\mathrm{x}, \mathrm{y})}{\frac{1}{\mathrm{MN}} \sum_{\mathrm{x}=1}^{\mathrm{y}=1} \sum_{\mathrm{x}=1}^{\mathrm{y}=1} \mathrm{I}^{\mathrm{b}}(\mathrm{x}, \mathrm{y})}
$$

7.VR module : In order to produce a high quality haze free image captured indifferent environments.we combines the information provided via the DCP module and CA modules to effectively recover the scene radiance is given by

$$
J^{C}(x, y)=\frac{\gamma^{C}\left(I^{c}(x, y)-A^{c}\right)}{\max \left(t_{h}(x, y), t_{0}\right)}+A^{c}+\sigma^{C}\left(\gamma^{C}-1\right)
$$

First, the intensity statistics of the RGB color chanel of the captured image can be calculated for the acquistion o color information via the probability mass function which is described as

$$
\operatorname{PMF},\left(\mathrm{I}_{\mathrm{k}}^{\mathrm{c}}\right)=\frac{\mathrm{n}_{\mathrm{k}}^{\mathrm{c}}}{\mathrm{MN}} \text { for } \mathrm{k}=0,1 \ldots \mathrm{L}
$$

\section{EXPERIMENTAL RESULTS}

The objective of this section is to demonstrate the results of proposed method and existing method comparisons. The below fig 3 shows that the existing method output results. The input hazy image is in applied with DCP module algorithm. Fig 4 shows that the proposed method de hazing and low wavelength compensation algorithm is used. In order to prove that the proposed method is an effective image restoration technique for images captured in wide range of weather conditions. So this algorithm is simple and restores the image quickly.
As mentioned in the introduction of this paper, the main aim of this research work is to provide an efficient algorithm for haze removal in images of practical usage. We have captured images in different inclined conditions for our evaluation on which our proposed algorithm as well as the existing algorithm applied.

The first image Fig1 (a) is an image captured on highway which is covered with fog on very busy hours of a working day. Here in Fig2 (a) we have considered an image captured when the road is cover by fog for our evaluation purpose. Both the images are processed under the algorithms and the output haze free restored images are presented herein this paper for observation. Different parameters such as PSNR (Peak Signal to Noise Ratio), RMSE (Root Mean Square Error) are calculated and the same are tabulated for our reference.

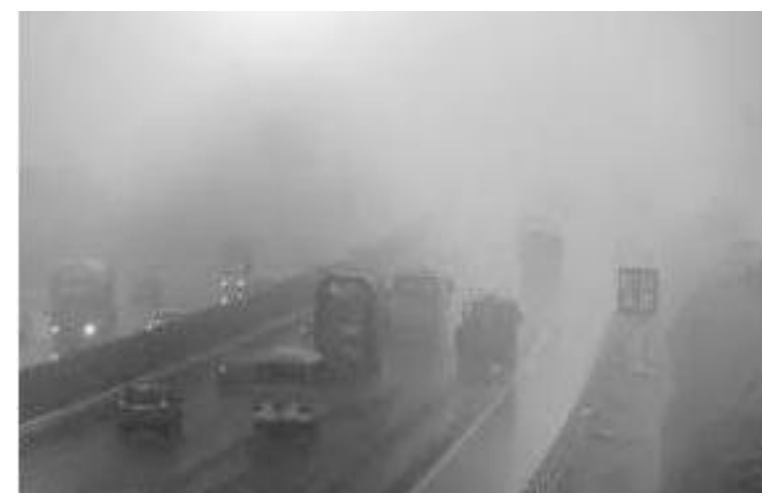

Fig 1(a):Input hazy image

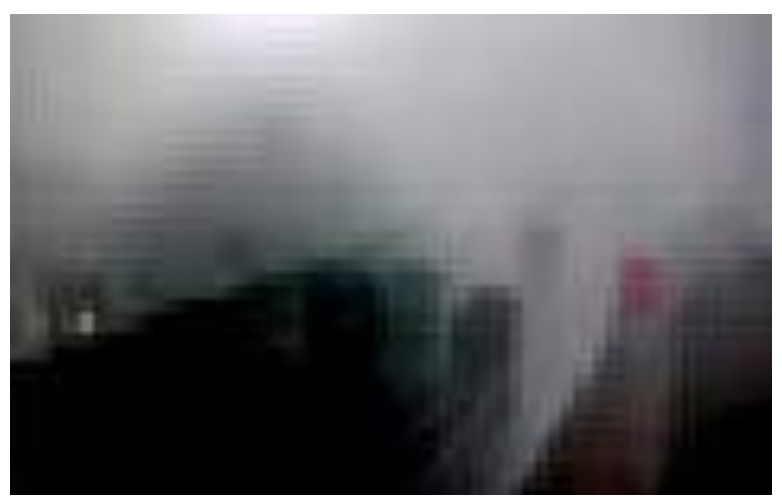

Fig1 (b): Haze free image (Existing method)

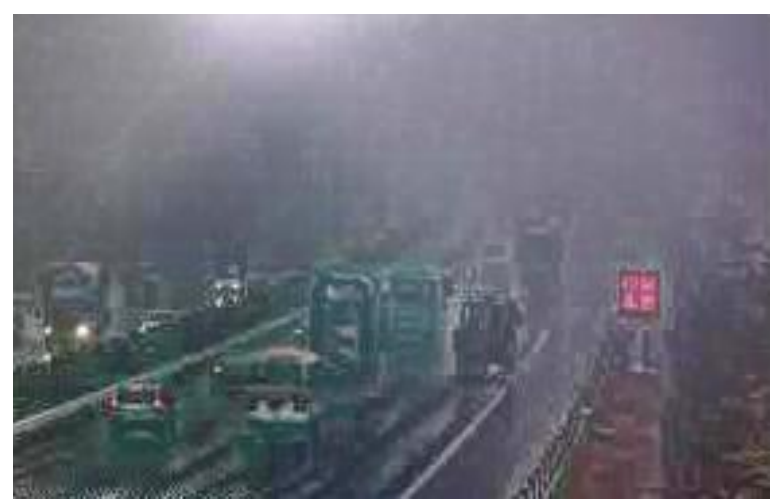

Fig1 (c): Haze free image (Proposed method) 


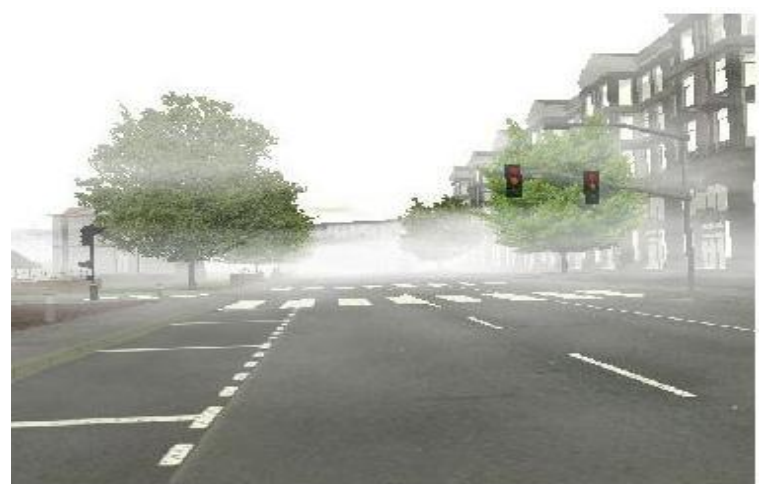

Fig2 (a): Input hazy Image

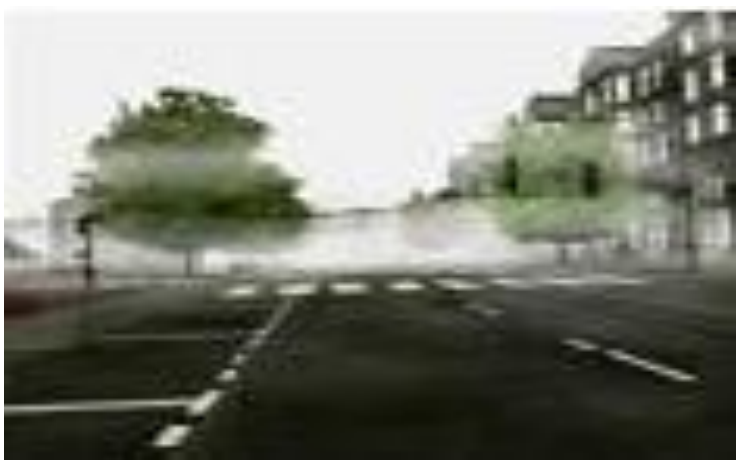

Fig2 (b): Haze free image (Existing)

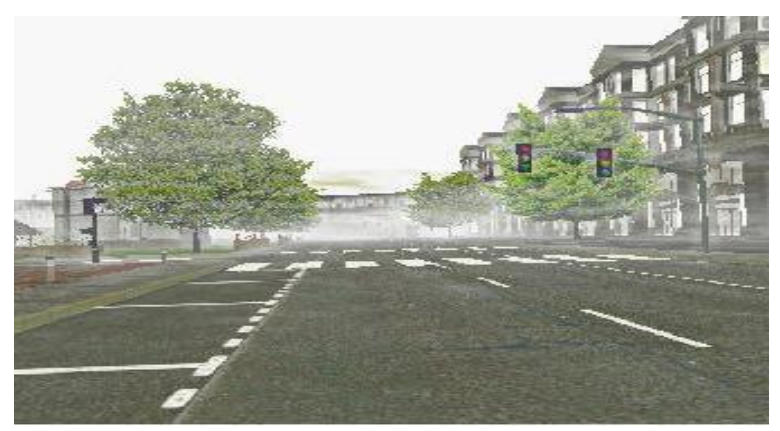

Fig2 (c): Haze free image (Proposed method)

The parameters used in table formulated are

PSNR: The peak signal to noise ratio the measure of restored image originality. It is measured in $\mathrm{dBs}$

$$
\text { PSNR }=10 \cdot \log _{10}\left(\frac{\mathrm{MAX}_{\mathrm{I}}^{2}}{\mathrm{MSE}}\right)
$$

RMSE: The RMSD serves to aggregate the magnitudes of the errors in predictions. $\hat{\theta}$ and $\theta$ are deviated and actual values.

E metric: For the non reference method, the e metric detects the rate of the restored visible edges in restored image. This can be expressed as

$$
\mathrm{e}=\frac{\mathrm{n}_{\mathrm{r}}-\mathrm{n}_{\mathrm{o}}}{\mathrm{n}_{\mathrm{o}}}
$$

Wheren $n_{r}$ and $n_{o}$ are the numbers of visible edges inthe restored haze free image and the original hazy image.

Correlation Coefficient (CC): The correlation coefficient is a number that summarizes the direction and degree (closeness) of linear relations between two variables.
The below presented table represents the results for existing and proposed methods of different parameters for both fig1 and fig 2 .

\begin{tabular}{|l|l|l|l|l|}
\hline \multirow{2}{*}{ Parameter } & \multicolumn{2}{|c|}{ Existing method } & \multicolumn{2}{c|}{$\begin{array}{c}\text { Proposed } \\
\text { method }\end{array}$} \\
\cline { 2 - 5 } & Fig1(b) & Fig2(b) & Fig1(c) & Fig2(c) \\
\hline Emetric & 0.5 & 0.5 & 0.5 & 0.5 \\
\hline R & 1.0037 & 1.0002 & 1.002 & 1.002 \\
\hline $\begin{array}{l}\text { PSNR } \\
\text { Value }\end{array}$ & 58.7309 & 59.6509 & 62.2552 & 68.9018 \\
\hline RMSE & 0.5132 & 0.5132 & 0.3420 & 0.1591 \\
\hline $\begin{array}{l}\text { Correlation } \\
\text { coefficient }\end{array}$ & 0.9883 & 0.9883 & 0.8789 & 0.9616 \\
\hline
\end{tabular}

Table 1: Comparison of results

\section{CONCLUSION}

The hazy image is suffers from low contrast and resolution due to poor visibility conditions. The hazy image enhancement using low complexity wavelength compensation algorithm is used to enhance haze image by de hazing algorithm. In this project influence of the atmospheric light is removed from the input hazy image. If the presence of atmospheric light is detected the influence of it is eliminated from the hazed input image. The haze effect can be removed by using WCID algorithm. The low complexity is achieved by using a median filter in the dark channel prior algorithm.

\section{REFERENCES}

[1] Wang, "Single image visibility enhancement in]Shih-chia Huang, Bo-Hao Chen, and Yi-Jui Cheng "An efficient visibility enhancement algorithm for road scenes captured by intelligent transportation systems" IEEE transactions on intelligent transportations systems 2014.

[2] Della Raju, Jaini Sara Babu "Removal of artificial light source and image dehazing in under water images using WCID algorithm" IEEE transactions on ISSN 2275-0181 Vol 3 Issue-3, march 2014.

[3] Y.J. Cheng, B.H. Chen, S.C. Huang, "A novel visibility restoration algorithm for single hazy images"Proc. IEEE Int. Conf. Syst., Man, Cybern., Oct.2013, pp.847-851.

[4] Y.J. Cheng, B.H. Chen, S.C. Huang, S.Y. Kuo, A.Kopylov, O.Seredin, Y. Vizilter, L.Mestetskiy, B.Vishnyakov, O.Vygolov, C.R.Lian, and C.T.Wu, "Visibility enhancement of single hazy images using hybrid dark channel prior," in Proc.IEEE Int. Syst.,Man,Cybern.,Oct.2013,pp.3627-3632.

[5] W.L. Li, B. Gu, J.T. Huang, S.Y. Wang, and M.Hgradientdomain,'IET Image Process.,vol.6, no.5,pp.589595,Jul.2012.

[6] K.He, J.Sun, and x.Tang, "Single image haze removal using dark channel prior," IEEE Trans. Pattern Anal.Mach.Intell., vol.33,no.12,pp.2341-2353,dec.2011.

[7] B.Xie, F.Guo, and Z.Cai,'Improved single image dehazing using dark channel prior and multi scalere tinex," in Proc.Int.Conf.Intell.Syst.Des.Eng.Appl.,Oct.2010,pp.848-851.

[8] R.Fattal "Single image dehazing," in Proc.ACM SIGGRAPH,2008,p.72.

[9] R.Tan, "Visibility in bad weather from a single image," in Proc.IEEEConf.Comput.Vis. Pattern Recognit., Jun.2008,pp.1-8.

[10] K. Tan and J.P.Oakley, "Enhancement of color images in poor visibility conditions,” Proc. ICIP, SEP. 2000, VOL.2, PP.788-7912 